\title{
Potential NRQED: The positronium case
}

\author{
A. Pineda* \\ Forschungszentrum Jülich, Institut für Kernphysik (Theorie), D-52425 Jülich, Germany \\ J. Soto ${ }^{\dagger}$ \\ Dept. d'Estructura i Constituents de la Matèria and IFAE, Universitat de Barcelona, Diagonal, 647, \\ E-08028 Barcelona, Catalonia, Spain
}

(Received 26 May 1998; published 7 December 1998)

\begin{abstract}
We discuss in detail potential NRQED (pNRQED), a previously proposed effective field theory for ultrasoft photons. The pNRQED Lagrangian for the equal mass case is presented, and it is shown that it correctly reproduces the positronium spectrum at order $m \alpha^{5}$. The pNRQED Lagrangian for the unequal mass case is also presented at the same order. Dimensional regularization is used throughout. [S0556-2821(98)06423-6]

PACS number(s): 11.10.St, 12.20.Ds, 12.39.Hg, 36.10.Dr
\end{abstract}

\section{INTRODUCTION}

Nonrelativistic QED (NRQED) [1] is becoming increasingly popular for QED bound state calculations $[1,2,3,4]$. It has the advantage over traditional Bethe-Salpeter equations [5] that the nonrelativistic nature of the QED bound states is explicit, whereas relativistic and radiative corrections can be systematically incorporated by taking into account higher orders in the $1 / m$ expansion and by calculating the matching coefficients at higher order in $\alpha$, respectively. However, the NRQED Lagrangian still contains two dynamical scales, namely, the typical relative momentum in the bound state $\mathbf{p}$ $\sim m \alpha$ and the bound state energy $E \sim m \alpha^{2}$, which implies that the terms in the Lagrangian do not have a unique size. The leading size of each term is given by the next relevant scale p (soft) (except for the time derivative) and rules have been provided to estimate the subleading contributions due to the scale $E$ (ultrasoft) [6]. Nevertheless, it would be helpful for bound state calculations to have an effective field theory (EFT) where each term in the Lagrangian had a welldefined size. This EFT has proved to be quite elusive for some time $[7,8,9]$.

In Ref. [10] we proposed potential NRQED (pNRQED) as such an EFT and presented the form of its Lagrangian for positronium. In Ref. [11] we worked out pNRQED for hydrogenlike atoms and reproduced the Lamb shift in a very straightforward way. It is the aim of this paper to discuss pNRQED for positronium in greater detail and to show that it also allows one to reproduce the spectrum at order $m \alpha^{5}$, where all regions of momenta (hard, soft, and ultrasoft) contribute, very efficiently. We also illustrate how dimensional regularization helps in that.

pNRQED describes fermion-antifermion pairs with relative momentum of order $\mathbf{p}$ and energy of order $E$, and ultrasoft photons with energy and momentum of order $E$. This should be compared with NRQCD, which describes degrees of freedom (fermions and photons) with energy and momentum less than a certain cutoff $\mu$ such that $E, \mathbf{p} \ll \mu \ll m$. For-

\footnotetext{
*Email address: a.pineda@fz-juelich.de

†Email address: soto@ecm.ub.es
}

mally speaking, pNRQED has two UV cutoffs $\Lambda_{1}$ and $\Lambda_{2}$, where $E \ll \Lambda_{1} \ll \mathbf{p}$ is the cutoff for the energy of the fermions and for the energy and momentum of the ultrasoft photons, whereas $\mathbf{p} \ll \Lambda_{2} \ll m$ is the cutoff for the relative momentum of the fermion-antifermion system. In principle, we have some freedom to choose the relative importance between $\Lambda_{1}$ and $\Lambda_{2}$. We choose $\Lambda_{2}^{2} / m \ll \Lambda_{1}$, which guarantees that the UV behavior of the fermion propagators in pNRQED is that of the static ones.

pNRQED is obtained from NRQED by integrating out fermions and photons of energies and momenta of order $\mathbf{p}$ and photons of energies of order $E$ and momenta p. ${ }^{1}$ The pNRQED Lagrangian obtained is local in time but nonlocal in space (i.e., it has potential terms) and contains ultrasoft photons only. The size of each term becomes explicit once the Lagrangian is projected onto the one-electron-onepositron subspace of the Fock space and written in terms of a wave function field. This is due to the fact that in the latter representation the ultrasoft photon fields can be multipole expanded about the center of mass. Moreover, the calculations in pNRQED are very close to those in nonrelativistic quantum mechanics.

The practical way in which we integrate out degrees of freedom is by a matching procedure. We impose that twofermion Green functions and four-fermion Green functions (with an arbitrary number of ultrasoft photon legs) in NRQED be equal to those in pNRQED once both are expanded about the external fermion energies and (ultrasoft) photon energies and momenta. Dimensional regularization (DR) is used for both UV and IR divergences. Furthermore, we use static propagators, and hence the matching can be done to a given order in $1 / m$ and $\alpha$. This is justified because the fermion energies we are integrating out in loops are of order $m \alpha$ whereas the typical kinetic energy is $O\left(m \alpha^{2}\right)$.

\footnotetext{
${ }^{1}$ Some authors $[12,13]$ like to distinguish potential photons, i.e., photons with $k^{0} \sim E$ and $\mathbf{k} \sim \mathbf{p}$, from soft photons, i.e., photons with $k^{0} \sim \mathbf{p}$ and $\mathbf{k} \sim \mathbf{p}$. This distinction is quite irrelevant in our formulation since both potential and soft photons are integrated out at the same time when matching NRQED to pNRQED.
} 
The procedure above is similar to the matching between QED and NRQED as carried out in Refs. [10,14,15]. In that case scales $\sim m$ are integrated out and the matching reduces to calculations in QED, where $m$ is the only scale in the integrals. Here, the scales which are integrated out are $\sim \mathbf{p}$ and the matching reduces to calculations in NRQED where $\mathbf{p}$ is the only scale in the integrals. Hence, the potential terms in $\mathrm{pNRQED}$ play a role analogous to the Wilson coefficients in NRQED. Indeed, the former encode contributions due to physics at the scale $\mathbf{p}$ much in the same way as the latter do of physics at the scale $m$. At each matching step the nonanalytic behavior in the scale which is integrated out becomes explicit.

We organize the paper as follows. In Sec. II we describe the matching procedure between NRQED and pNRQED. In Sec. III we present the bound state calculation. Section IV is devoted to the conclusions and future prospects. In Appendix A the gauge independence is checked at order $m \alpha^{4}$ by calculating the matching to this order in the Feynman gauge. In Appendix B the pNRQED Lagrangian for the unequal mass case is displayed.

\section{MATCHING NRQED TO pNRQED}

The pieces of the NRQED Lagrangian which are relevant to the calculation of the bound state energy at $O\left(m \alpha^{5}\right)$ read

$$
\begin{aligned}
\mathcal{L}_{\mathrm{NRQED}}= & \psi^{\dagger}\left\{i D^{0}+\frac{\mathbf{D}^{2}}{2 m}+\frac{\mathbf{D}^{4}}{8 m^{3}}+c_{F} e \frac{\boldsymbol{\sigma} \cdot \mathbf{B}}{2 m}\right. \\
& \left.+c_{D} e \frac{[\boldsymbol{\nabla} \cdot \mathbf{E}]}{8 m^{2}}+i c_{S} e \frac{\boldsymbol{\sigma} \cdot(\mathbf{D} \times \mathbf{E}-\mathbf{E} \times \mathbf{D})}{8 m^{2}}\right\} \psi \\
& +\left(\chi_{c}, e \rightarrow-e\right)-\frac{d_{s}}{m^{2}} \psi^{\dagger} \psi \chi_{c}^{\dagger} \chi_{c} \\
& +\frac{d_{v}}{m^{2}} \psi^{\dagger} \boldsymbol{\sigma} \psi \chi_{c}^{\dagger} \boldsymbol{\sigma} \chi_{c}-\frac{1}{4} F_{\mu \nu} F^{\mu \nu}+\frac{d_{2}}{m^{2}} F_{\mu \nu} D^{2} F^{\mu \nu},
\end{aligned}
$$

where $\psi$ is the Pauli spinor field that annihilates the fermion and $\chi_{c}$ is the Pauli spinor field that annihilates the antifermion. $^{2} i D^{0}=i \partial_{0}-e A^{0}, i \mathbf{D}=i \boldsymbol{\nabla}+e \mathbf{A}$ on the fermion field. The bilinear Lagrangian for $\chi_{c}$ is equal to the $\psi \mathrm{La}-$ grangian with change $e \rightarrow-e$.

This can be seen as follows. We draw all possible diagrams of the two-fermion-irreducible four-fermion Green function, which cannot be disconnected by cutting a photon line, such that $s+r \leqslant 4$, where $s$ is the number of $1 / m$ factors in the diagram and $r$ the number of explicit $\alpha[6,10,11]$. This rule can be easily justified if we take into account that the next relevant scale is $\mathbf{p} \sim m \alpha$ and hence all $1 / m$ must be compensated for by $m \alpha$ until we reach dimensions of energy. For diagrams which can be disconnected by cutting a

\footnotetext{
${ }^{2}$ We use here $\chi_{c}=C \chi^{*}$, where $C$ is the charge conjugation matrix, instead of $\chi$ as in Refs. [10,11] because it is more usual in nonrelativistic systems.
}

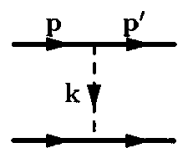

(a)

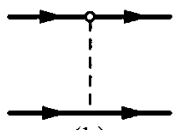

(b)

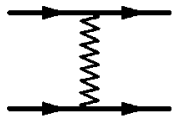

(e)

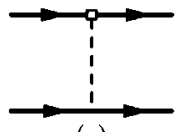

(c)

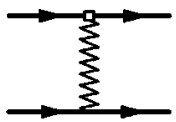

(f)

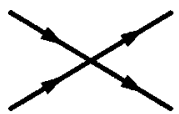

(h)

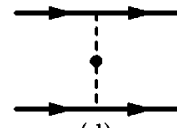

(d)

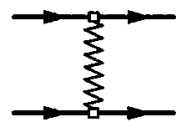

(g)
FIG. 1. The nonzero relevant diagrams for the matching at the tree level in the Coulomb gauge. The dashed and zigzag lines represent the $A_{0}$ and $\mathbf{A}$ fields, respectively, while the solid lines represent the fermion and antifermion fields. The first diagram is the Coulomb potential. For $A_{0}$ the open circle is the vertex proportional to $c_{D}$, the square to $c_{S}$ (spin dependent), and the solid circle to $d_{2}$ (the vacuum polarization), while for $\mathbf{A}$ the square is the vertex proportional to $c_{F}$ and the other vertex appears from the covariant derivative in the kinetic term. The last diagram is proportional to $d_{s}$ and $d_{v}$. The symmetric diagrams are not displayed.

photon line the same rule applies but there is an extra suppression if $n$ time derivatives act on this photon line. This is due to the fact that these time derivatives are only sensitive to the typical energy. The extra suppression factor is $\alpha^{n}$. Recall also that any diagram may have subleading contributions which appear from the analytic expansion of the external energy about zero. In the Coulomb gauge the diagrams satisfying the above criteria are displayed in Figs. 1 and 2. Then both tree-level and one-loop diagrams are required. Notice also that $c_{F}, c_{D}$, and $c_{S}$ are needed at order $\alpha$ and $d_{s}$ and $d_{v}$ at order $\alpha^{2}$. All these Wilson coefficients are gauge independent but depend on the renormalization scheme for the UV divergences of NRQED. We shall use dimensional regularization with the modified minimal subtraction $(\overline{\mathrm{MS}})$ scheme. In this scheme the Wilson coefficients of the bilinear terms were given in [14] and the ones for the fourfermion terms in $[10,15]$. They read

$$
\begin{aligned}
& c_{F}=1+\frac{\alpha}{2 \pi}, \\
& c_{D}=1+\frac{\alpha}{\pi}\left(\frac{4}{3} \log \frac{m^{2}}{\mu^{2}}\right),
\end{aligned}
$$

$$
c_{S}=1+\frac{\alpha}{\pi},
$$




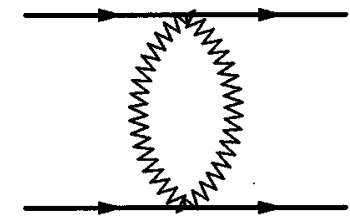

(a)

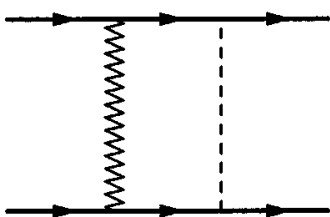

(b)

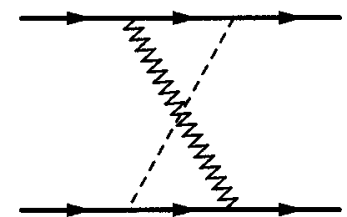

(c)
FIG. 2. The nonzero relevant diagrams for the matching at one loop in the Coulomb gauge. The dashed and zigzag lines represent the $A_{0}$ and $\mathbf{A}$ fields, respectively, while the solid lines represent the fermion and antifermion. The interactions for $\mathbf{A}$ are the ones which appear from the covariant space derivatives in the kinetic term, while for $A_{0}$ they come from the covariant time derivative. The symmetric diagrams are not displayed.

$$
\begin{aligned}
& d_{2}=\frac{\alpha}{60 \pi} \\
& d_{s}=\frac{3 \pi \alpha}{2}\left\{1-\frac{2 \alpha}{3 \pi}\left(\log \frac{m^{2}}{\mu^{2}}+\frac{23}{3}-\log 2+i \frac{\pi}{2}\right)\right\}, \\
& d_{v}=-\frac{\pi \alpha}{2}\left\{1-\frac{2 \alpha}{\pi}\left(\frac{22}{9}+\log 2-i \frac{\pi}{2}\right)\right\} .
\end{aligned}
$$

Hence our starting point is the Lagrangian (2.1) with the Wilson coefficients (2.2). We wish to integrate out fermions and photons of energy and momenta $\sim \mathbf{p}$ and photons of energy $\sim E$ and momentum $\sim \mathbf{p}$. Then the effective theory we want to reach, namely, pNRQED, contains only ultrasoft photons (of energy and momentum $\sim E$ ) and fermions of energy $\sim E$ and momentum $\sim \mathbf{p}$ or less. Since we integrate out photon momenta $\sim \mathbf{p}$ but keep fermion momenta of this order, pNRQED contains terms nonlocal in space, namely, potential terms. This is not a problem for a nonrelativistic EFT.

The practical way to obtain pNRQED is by enforcing two- and four-fermion Green functions with arbitrary ultrasoft external photons to be equal to those of NRQED once we expand about zero the energy in the external electron legs and the energy and momenta of the ultrasoft photon legs. This may produce IR divergences which are regulated in dimensional regularization, in the same way as the UV divergences are. Since the IR behavior of NRQED and pNRQED is the same, these IR divergences will cancel out in the matching. The UV divergences of NRQED must be renormalized in the $\overline{\mathrm{MS}}$ if we want to use the matching coefficients (2.2). We still have a choice in the renormalization scheme of pNRQED. However, it is most advantageous to use again $\overline{\mathrm{MS}}$. Indeed, in this scheme we can blindly use $\overline{\mathrm{MS}}$ for any divergence regardless of whether it is UV or IR in the matching calculation. For the UV divergences of NRQED and pNRQED it is just the scheme we choose, and for the IR divergences it is irrelevant as long as we use the same treatment in both theories, since the IR behavior is the same. This allows us to put integrals with no scale equal to zero and, as we will see later on, reduces the matching to a calculation in NRQED only.

Notice that we demand off-shell Green functions in NRQED and pNRQED to be equal and not on-shell Green functions as is customary in the matching from QED to NRQED [1,14]. This is due to the fact that we are eventually interested in bound states, and particles in a bound state are typically off shell. The equations of motion of pNRQED (with potential terms included), or local field redefinitions, may be consistently used later on to remove time derivatives in higher order terms and write the pNRQED Lagrangian in a standard form, within the philosophy advocated in Ref. [16] (see also [17]). We have checked in Appendix A that this procedure produces gauge-independent results at $O\left(m \alpha^{4}\right) .^{3}$ In fact the same argument applies to the matching between QED and NRQED, which accordingly should also be carried out off shell. However, in that case, at lower orders there is no difference between doing the matching on shell and replacing derivatives by covariant derivatives, in order to enforce gauge invariance, and doing the matching off shell and consistently using the equations of motion or local field redefinitions to get rid of time derivative terms $[14,16]$.

The remaining important ingredient to carry out the matching efficiently is the use of static [heavy quark effective theory (HQET)] propagators for the fermions. This has been completely justified in the matching between QED and NRQED $[14,15]$, since both energy and momentum in this theory cannot exceed the same cutoff $\Lambda$ which is smaller than $m$. Hence the UV behavior of the fermion propagator in NRQED is always dominated by the energy. This fact is not automatically implemented in dimensional regularization. When dimensional regularization is used, the correct UV behavior of NRQED is only obtained when expanding about the static propagator.

In pNRQED we have a certain choice for the UV cutoffs for the fermion energy and momentum. We shall choose $\Lambda_{1} \ll \mathbf{p}$ for the energy and $\Lambda_{2} \ll m$ for the momentum in such a way that $\Lambda_{2}^{2} / m \ll \Lambda_{1}$. The proper way to implement this condition in dimensional regularization is again by expanding the fermion propagator in pNRQED about the static propagator.

Now we are in a position to prove that no pNRQED diagram containing a loop contributes to the matching calculation.

\footnotetext{
${ }^{3}$ However, there is still some freedom in the choice of the wave function field, to be introduced later on, due to time-independent unitary transformations which commute with the leading terms in the pNRQED Lagrangian. Therefore, in general, it is not to be expected that the standard forms of the pNRQED Lagrangian calculated with different gauges coincide, but only to be related by one such unitary transformation. This explains, for instance, why the potential presented in [19] is different from ours but leads to the same physics.
} 
Consider first the two-fermion Green function with an arbitrary number of ultrasoft legs. For potential terms to contribute we need at least a four-fermion Green function and hence we only have to care about ultrasoft photons. If we input a momentum $\sim \mathbf{p}$ in the fermion line, this momentum cannot flow out through any external ultrasoft photon line (by definition of ultrasoft). Then it must flow through the fermion line, which is a series of static propagators insensible to the momentum flowing. Hence upon expanding about external fermion energies and external energies and momenta of the ultrasoft photons there is no scale in any integral and therefore any loop contribution vanishes. In fact, exactly the same argumentation can be used for the NRQED calculation. Then we conclude that terms bilinear in fermions are exactly the same in NRQED and pNRQED. However, we have to keep in mind that the latter (by definition) must be understood as containing ultrasoft (US) photons only.

Consider next the four-fermion Green function in pNRQED containing several potential terms but no US photon. Since no energy can flow through the potentials and the static propagators are insensitive to the momentum, upon expanding about the US external energy, the integrals over internal energies have no scale. However, these integrals have IR (pinch) singularities which are not regulated by standard DR. We shall take the additional prescription of putting them to zero. Since the IR behavior of pNRQED and NRQED is the same, the same kind of integrals appear in the NRQED calculation. If we put them consistently to zero, we obtain the correct potential terms, which play a role similar to the Wilson coefficients in the matching between QED and NRQED. It is important to keep in mind that the Wilson coefficients compensate for the different UV behavior of the effective theory (pNRQED) with respect to that of the "fundamental" theory (NRQED). Hence they are not sensitive to the details of the IR behavior, which legitimates the prescription above. Then any loop diagram in pNRQED with no US photons can be put to zero. This still holds if an arbitrary number of US photon lines are included in the diagram. Indeed, any potential line in the diagram now may also contain US momenta from the photon lines. These, however, can be expanded about zero since they are (by definition) much smaller than the momentum transfer in the potential. Hence the integrals over US photon energies and momenta contain no scale (again upon expanding the US external energy in the fermion static propagators) and can also be put to zero.

In summary, we can directly identify the potential terms from a calculation in NRQED. We would like to stress again the similarity in the procedure with the matching between QED and NRQED as carried out in Refs. [14,15]. The potential terms in $\mathrm{PNRQED}$ play the role of Wilson coefficients in the matching procedure.

The four-fermion terms appearing in the pNRQED Lagrangian typically have the form ${ }^{4}$

\footnotetext{
${ }^{4}$ In principle, Eq. (2.3) could also depend on the total momentum $\mathbf{P}=-i \boldsymbol{\nabla}_{\mathbf{X}}$, with $\mathbf{X}=\left(\mathbf{x}_{1}+\mathbf{x}_{2}\right) / 2$, or on ultrasoft photons, but these effects can be neglected to the accuracy we are working at.
}

$$
\begin{aligned}
L^{\mathrm{pot}}= & -\int d^{3} \mathbf{x}_{1} d^{3} \mathbf{x}_{2} \psi^{\dagger}\left(t, \mathbf{x}_{1}\right) \chi_{c}^{\dagger}\left(t, \mathbf{x}_{2}\right) V\left(\mathbf{x}, \mathbf{p}, \boldsymbol{\sigma}_{1}, \boldsymbol{\sigma}_{2}\right) \\
& \times \chi_{c}\left(t, \mathbf{x}_{2}\right) \psi\left(t, \mathbf{x}_{1}\right),
\end{aligned}
$$

where $\mathbf{x}=\mathbf{x}_{1}-\mathbf{x}_{2}, \mathbf{p}=-i \nabla_{\mathbf{x}}$ and $s_{1}=\boldsymbol{\sigma}_{1} / 2, s_{2}=\boldsymbol{\sigma}_{2} / 2$ act on the fermion and antifermion, respectively (the spin fermion and antifermion indices are contracted with the potential indices, which are not explicitly displayed). $V\left(\mathbf{x}, \mathbf{p}, \boldsymbol{\sigma}_{1}, \boldsymbol{\sigma}_{2}\right)$ may also be written as an expansion of the type

$$
V=V^{(0)}+V^{(1)}+V^{(2)}+V^{(3)}+\cdots,
$$

where $\left\langle V^{(n)}\right\rangle \sim m \alpha^{n}$. Our results are exact for the four first terms of this expansion.

We obtain, from the tree-level diagrams of Fig. 1 ( $\widetilde{V}$ represents the Fourier transform of $V$ ),

$$
\begin{aligned}
& \tilde{V}_{\text {tree }}^{(a)}=-\frac{4 \pi \alpha}{\mathbf{k}^{2}}, \\
& \tilde{V}_{\text {tree }}^{(b)}=\frac{\pi \alpha c_{D}}{m^{2}}, \\
& \tilde{V}_{\text {tree }}^{(c)}=-\frac{i 2 \pi \alpha c_{S}}{m^{2}} \frac{(\mathbf{p} \times \mathbf{k}) \cdot \mathbf{S}}{\mathbf{k}^{2}}, \\
& \tilde{V}_{\text {tree }}^{(d)}=-\frac{16 \pi \alpha d_{2}}{m^{2}}, \\
& \tilde{V}_{\text {tree }}^{(e)}=-\frac{4 \pi \alpha}{m^{2}}\left(\frac{\mathbf{p}^{2}}{\mathbf{k}^{2}}-\frac{(\mathbf{p} \cdot \mathbf{k})^{2}}{k^{4}}\right), \\
& \tilde{V}_{\text {tree }}^{(f)}=-\frac{i 4 \pi \alpha c_{F}}{m^{2}} \frac{(\mathbf{p} \times \mathbf{k}) \cdot \mathbf{S}}{\mathbf{k}^{2}}, \\
& \tilde{V}_{\text {tree }}^{(g)}=\frac{4 \pi \alpha c_{F}^{2}}{m^{2}}\left(\mathbf{s}_{1} \cdot \mathbf{s}_{2}-\frac{\mathbf{s}_{1} \cdot \mathbf{k} \mathbf{s}_{2} \cdot \mathbf{k}}{\mathbf{k}^{2}}\right), \\
& \tilde{V}_{\text {tree }}^{(h)}=\left(d_{s}+3 d_{v}\right)-2 d_{v} \mathbf{S}^{2},
\end{aligned}
$$

where $\mathbf{S}=s_{1}+s_{2}$, and, for the one-loop diagrams of Fig. 2,

$$
\begin{aligned}
& \widetilde{V}_{1 \text { loop }}^{(a)}=\frac{\alpha^{2}}{m^{2}}\left(\log \frac{\mathbf{k}^{2}}{\mu^{2}}-\frac{8}{3} \log 2+\frac{5}{3}\right), \\
& \widetilde{V}_{1 \text { loop }}^{(b, c)}=\frac{4 \alpha^{2}}{3 m^{2}}\left(\log \frac{\mathbf{k}^{2}}{\mu^{2}}+2 \log 2-1\right) .
\end{aligned}
$$

The $\mu$ dependence of Figs. $2 \mathrm{~b}, 2 \mathrm{c}$ is of IR origin and will eventually cancel with US contributions. However, the $\mu$ dependence of Fig. 2a is of UV origin and cancels exactly with the $\mu$ dependence of $d_{s}$. Recall that there is an additional $\mu$ dependence in $c_{D}$ which will also cancel against US contributions. Upon Fourier transforming and putting together the above results we obtain 


$$
V=V^{(0)}+\delta V
$$

where

$$
V^{(0)}=-\frac{\alpha}{|\mathbf{x}|}
$$

and

$$
\begin{aligned}
\delta V= & -\frac{\alpha}{2 m^{2}} \frac{1}{|\mathbf{x}|}\left(\mathbf{p}^{2}+\frac{1}{\mathbf{x}^{2}} \mathbf{x} \cdot(\mathbf{x} \cdot \mathbf{p}) \mathbf{p}\right)+\frac{\delta^{(3)}(\mathbf{x})}{m^{2}}\left(\pi \alpha\left(c_{D}-2 c_{F}^{2}\right)+d_{s}+3 d_{v}-16 \pi \alpha d_{2}+\frac{\alpha^{2}}{3}-\frac{7 \alpha^{2}}{3} \log \mu^{2}\right) \\
& -\frac{7 \alpha^{2}}{6 \pi m^{2}} \operatorname{reg} \frac{1}{|\mathbf{x}|^{3}}+\frac{\delta^{(3)}(\mathbf{x})}{m^{2}} \mathbf{S}^{2}\left(\pi \alpha \frac{4}{3} c_{F}^{2}-2 d_{v}\right)+\frac{\alpha}{4 m^{2}} \frac{1}{|\mathbf{x}|^{3}} \mathbf{L} \cdot \mathbf{S}\left(2 c_{S}+4 c_{F}\right)+\frac{\alpha c_{F}^{2}}{4 m^{2}} \frac{1}{|\mathbf{x}|^{3}} S_{12}(\hat{\mathbf{x}}),
\end{aligned}
$$

where $S_{12}(\hat{\mathbf{x}})=\left(-\boldsymbol{\sigma}_{1} \cdot \boldsymbol{\sigma}_{2}+3 \boldsymbol{\sigma}_{1} \cdot \hat{\mathbf{x}} \sigma_{2} \cdot \hat{\mathbf{x}}\right)$ and (see [18] for more details on the Fourier transform)

$$
-\frac{1}{4 \pi} \operatorname{reg} \frac{1}{|\mathbf{x}|^{3}}=\int \frac{d^{3} \mathbf{k}}{(2 \pi)^{3}} e^{i \mathbf{k} \cdot \mathbf{x}} \log k .
$$

The pNRQED Lagrangian now reads

$$
\begin{aligned}
L_{\mathrm{pNRQED}}= & \int d^{3} \mathbf{x}\left(\psi^{\dagger}\left\{i D^{0}+\frac{\mathbf{D}^{2}}{2 m}+\frac{\mathbf{D}^{4}}{8 m^{3}}\right\} \psi+\left(\chi_{c}, e \rightarrow-e\right)-\frac{1}{4} F_{\mu \nu} F^{\mu \nu}\right) \\
& -\int d^{3} \mathbf{x}_{1} d^{3} \mathbf{x}_{2} \psi^{\dagger}\left(t, \mathbf{x}_{1}\right) \chi_{c}^{\dagger}\left(t, \mathbf{x}_{2}\right) V\left(\mathbf{x}, \mathbf{p}, \boldsymbol{\sigma}_{1}, \boldsymbol{\sigma}_{2}\right) \chi_{c}\left(t, \mathbf{x}_{2}\right) \psi\left(t, \mathbf{x}_{1}\right),
\end{aligned}
$$

where the photons are ultrasoft.

In order to make explicit the size of each term in Eq. (2.19) it is convenient to project pNRQED to the oneelectron-one-position subspace (this can be easily done at the Hamiltonian level). This subspace is spanned by

$$
\int d^{3} \mathbf{x}_{1} d^{3} \mathbf{x}_{2} \varphi\left(\mathbf{x}_{1}, \mathbf{x}_{2}\right) \psi^{\dagger}\left(\mathbf{x}_{1}\right) \chi_{c}^{\dagger}\left(\mathbf{x}_{2}\right)|0\rangle
$$

where $|0\rangle$ is the subspace of the Fock space containing zero electrons and positrons, but an arbitrary number of ultrasoft photons.

Then the dynamics of the wave function field is described by the Lagrangian

$$
\begin{aligned}
L_{\mathrm{PNRQED}}= & \int d^{3} \mathbf{x}_{1} d^{3} \mathbf{x}_{2} \varphi^{\dagger}\left(\mathbf{x}_{1}, \mathbf{x}_{2}, t\right) \\
& \times\left(i \partial_{0}+\frac{\boldsymbol{\nabla}_{\mathbf{x}}^{2}}{m}+\frac{\boldsymbol{\nabla}_{\mathbf{X}}^{2}}{4 m}+\frac{\boldsymbol{\nabla}_{\mathbf{x}}^{4}}{4 m^{3}}-e x^{i} \partial_{i} A_{0}(t, \mathbf{X})\right. \\
& \left.-2 i e \frac{\mathbf{A}(t, \mathbf{X}) \cdot \boldsymbol{\nabla}_{\mathbf{x}}}{m}-V\left(\mathbf{x}, \mathbf{p}, \boldsymbol{\sigma}_{1}, \boldsymbol{\sigma}_{2}\right)\right) \\
& \times \varphi\left(\mathbf{x}_{1}, \mathbf{x}_{2}, t\right)-\int d^{3} \mathbf{x} \frac{1}{4} F_{\mu \nu} F^{\mu \nu}
\end{aligned}
$$

where we have made precise that the remaining photon fields are ultrasoft by multipole expanding them about the center of mass. ${ }^{5}$ Furthermore, gauge invariance at any order in the multipole expansion can be made explicit by introducing

$$
\varphi\left(\mathbf{x}_{1}, \mathbf{x}_{2}, t\right)=P\left[\exp \left(i e \int_{\mathbf{x}_{2}}^{\mathbf{x}_{1}} \mathbf{A} \cdot d \mathbf{x}\right)\right] S(\mathbf{x}, \mathbf{X}, t) .
$$

Then, the gauge transformations of the above wave function fields are

$$
\begin{gathered}
\varphi\left(\mathbf{x}_{1}, \mathbf{x}_{2}, t\right) \rightarrow g\left(\mathbf{x}_{1}, t\right) \psi\left(\mathbf{x}_{1}, \mathbf{x}_{2}, t\right) g^{-1}\left(\mathbf{x}_{2}, t\right), \\
S(\mathbf{x}, \mathbf{X}, t) \rightarrow S(\mathbf{x}, \mathbf{X}, t) .
\end{gathered}
$$

We finally obtain

$$
\begin{aligned}
L_{\mathrm{PNRQED}}= & \int d^{3} \mathbf{x} d^{3} \mathbf{X} d t S^{\dagger}(\mathbf{x}, \mathbf{X}, t)\left\{i \partial_{0}-\frac{\mathbf{p}^{2}}{m}+\frac{\mathbf{p}^{4}}{4 m^{3}}-\frac{\mathbf{P}^{2}}{4 m}\right. \\
& \left.-V\left(\mathbf{x}, \mathbf{p}, \boldsymbol{\sigma}_{1}, \boldsymbol{\sigma}_{2}\right)+e \mathbf{x} \cdot \mathbf{E}(\mathbf{X}, t)\right\} \\
& \times S(\mathbf{x}, \mathbf{X}, t)-\int d^{3} \mathbf{x} \frac{1}{4} F_{\mu \nu} F^{\mu \nu},
\end{aligned}
$$

which is explicitly gauge invariant. Moreover, the size of each term is unique and can be evaluated as follows. Each

\footnotetext{
${ }^{5}$ As expected for a chargeless particle, the covariant derivatives for $\mathbf{P}$ are the ordinary ones.
} 
relative momentum $\partial_{\mathbf{x}}$ and inverse relative coordinate $|\mathbf{x}|^{-1}$ have a size $\sim m \alpha$. Each US photon field, derivatives acting on it, the time derivative, and the center-of-mass momentum $\partial_{\mathbf{X}}$ (in the rest frame, when entering in recoil corrections due to the virtual emission of US photons) on the wave function field have a size $\sim m \alpha^{2}$. Formula (2.23) has already been presented in [10] (except for a numerical factor in the potential which was left arbitrary). We shall use it to calculate the spectrum at $O\left(m \alpha^{5}\right)$ in the next section.

The gauge independence of the matching calculation is checked at $O\left(m \alpha^{4}\right)$ in Appendix A. The pNRQED Lagrangian for the unequal mass case can be built with no further difficulty. The result is displayed in Appendix B.

\section{BOUND STATE CALCULATION IN pNRQED}

In order to find the corrections to the bound state energy of a state with principal quantum number $n$ we consider the Green function (we will follow a procedure and notation similar to Ref. [11])

$$
\Pi(q, \mathbf{x}):=\int d x^{0} d \mathbf{X} e^{i q x^{0}}\left\langle T\left\{\varphi(0) \varphi^{\dagger}(\mathbf{x}, \mathbf{X}, t)\right\}\right\rangle
$$

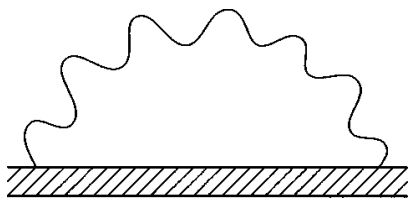

FIG. 3. The thick line and wavy lines are the positronium and the transverse ultrasoft photon propagators, respectively.

when $q \rightarrow E_{n}$, where $E_{n}$ is the energy of the leading Hamiltonian

$$
\hat{h}_{0}=-\frac{\nabla^{2}}{m}-\frac{\alpha}{|\mathbf{x}|} .
$$

The integral over $\mathbf{X}$ fixes the center-of-mass momentum $\mathbf{P}$ to zero. We write

$$
\Pi(q, \mathbf{x})=\frac{A_{n}+\delta A_{n}}{q-\left(E_{n}+\delta E_{n}\right)} \sim \frac{A_{n}+\delta A_{n}}{q-E_{n}}+\frac{A_{n}}{q-E_{n}} \delta E_{n} \frac{1}{q-E_{n}} .
$$

The contribution to $\delta E_{n}$ coming from the correction to the potential and the kinetic energy read

$$
\begin{aligned}
& \delta^{V} E_{n}=\langle n l j|\delta V| n l j\rangle= \frac{m \alpha^{4}}{8}\left\{-\frac{3}{n^{3}(2 l+1)}+\frac{1}{n^{4}}\right. \\
&-\frac{2 \alpha}{3 \pi} \frac{\delta_{l 0}}{n^{3}}\left[-\log \frac{m}{\mu}+7 \log \frac{\mu n}{m \alpha}-6 \log 2+\frac{17}{5}-7\left(\sum_{k=1}^{n} \frac{1}{k}+\frac{n-1}{2 n}\right)\right]-\frac{7 \alpha}{3 \pi} \frac{1-\delta_{l 0}}{n^{3}} \frac{1}{l(l+1)(2 l+1)} \\
&\left.+\frac{14}{3} \frac{\delta_{l 0} \delta_{s 1}}{n^{3}}\left[1+\frac{3 \alpha}{7 \pi}\left(-\frac{32}{9}-2 \log 2\right)\right]+\frac{\left(1-\delta_{l 0}\right) \delta_{s 1}}{l(2 l+1)(l+1) n^{3}} C_{j, l}\right\} \\
& \delta^{K} E_{n}=-\frac{1}{4 m^{3}}\left\langle n l j\left|\nabla^{4}\right| n l j\right\rangle=\frac{m \alpha^{4}}{8} \frac{3(l+1 / 2)-4 n}{4 n^{4}(2 l+1)}
\end{aligned}
$$

where

$$
C_{j, l}= \begin{cases}-\frac{l+1}{2 l-1}\left(2(3 l-1)+\frac{\alpha}{\pi}(4 l-1)\right), & j=l-1, \\ -2-\frac{\alpha}{\pi}, & j=l, \\ \frac{l}{2 l+3}\left(2(3 l+4)+\frac{\alpha}{\pi}(4 l+5)\right), & j=l+1 .\end{cases}
$$

There is also a contribution from a virtual exchange of an ultrasoft photon corresponding to the diagram in Fig. 3, which has already been evaluated in dimensional regularization for the hydrogenlike atom [11] (here the calculation is identical but using the reduced mass). Notice that the $\overline{\mathrm{MS}}$ scheme has to be used in the calculation. Since Eq. (2.24) is gauge invariant, we can use any gauge to calculate this contribution. Still the Coulomb gauge continues to be advantageous, since in this gauge $A_{0}$ can only contribute to tadpoles which can be safely put to zero in dimensional regularization. This contribution reads

$$
\begin{aligned}
\delta^{\mathrm{US}} E_{n} & =-\frac{8}{3} \frac{\alpha}{\pi} \sum_{m}\left|\left\langle n\left|\frac{\mathbf{p}}{m}\right| m\right\rangle\right|^{2}\left(E_{n}-E_{m}\right)\left(\log \frac{\mu}{\left|E_{n}-E_{m}\right|}-\log 2+\frac{5}{6}\right) \\
& =-\frac{m \alpha^{5}}{3 \pi n^{3}}\left(\delta_{l 0}\left[\log \frac{\left\langle E_{n, l}\right\rangle^{2}}{\mu^{2}}-\frac{5}{3}\right]+\left(1-\delta_{l 0}\right) \log \frac{4\left\langle E_{n, l}\right\rangle^{2}}{m^{2} \alpha^{4}}\right),
\end{aligned}
$$


where the last equation implicitly defines $\left\langle E_{n, l}\right\rangle$. Once we add all these contributions the final result reads

$$
\begin{aligned}
\delta E_{n, l, j}= & \delta^{V} E_{n}+\delta^{K} E_{n}+\delta^{\mathrm{US}} E_{n}=\frac{m \alpha^{4}}{8}\left\{-\frac{4}{n^{3}(2 l+1)}+\frac{11}{8 n^{4}}\right. \\
& -\frac{2 \alpha}{3 \pi} \frac{\delta_{l 0}}{n^{3}}\left[9 \log \alpha+7 \log n+8 \log R(n, l)-14 \log 2-\frac{49}{15}-7\left(\sum_{k=1}^{n} \frac{1}{k}+\frac{n-1}{2 n}\right)\right] \\
& -\frac{16 \alpha}{3 \pi} \frac{1-\delta_{l 0}}{n^{3}}\left(\log R(n, l)+\frac{7}{16} \frac{1}{l(l+1)(2 l+1)}\right)+\frac{14}{3} \frac{\delta_{l 0} \delta_{s 1}}{n^{3}}\left[1+\frac{3 \alpha}{7 \pi}\left(-\frac{32}{9}-2 \log 2\right)\right] \\
& \left.+\frac{\left(1-\delta_{l 0}\right) \delta_{s 1}}{l(2 l+1)(l+1) n^{3}} C_{j, l}\right\},
\end{aligned}
$$

where $\log R(n, l)=\log \left(2\left\langle E_{n, l}\right\rangle / m \alpha^{2}\right)$ is called the Bethe logarithm. For the $O\left(m \alpha^{5}\right)$ contribution we find agreement with Ref. [19] for the spin-independent piece and for the $\delta_{l 0} \delta_{s 1}$ piece, while for the $\left(1-\delta_{l 0}\right) \delta_{s 1}$ piece we find agreement with Ref. [3] (this last piece could also be obtained from results of Ref. [19]).

We can also easily obtain the full decay width at lowest order. It reads

$$
\Gamma_{n}=\frac{m \alpha^{5}}{2 n^{3}} \delta_{10}\left(1-\delta_{s 1}\right)+\sum_{m<n} \frac{16}{3} \alpha\left|\left\langle n\left|\frac{\mathbf{p}}{m}\right| m\right\rangle\right|^{2}\left(E_{n}-E_{m}\right) .
$$

\section{DISCUSSION AND CONCLUSIONS}

We have seen that $\mathrm{pNRQED}$ correctly reproduces the positronium spectrum at $O\left(m \alpha^{5}\right)$. This is a nontrivial check of the ideas behind this EFT since at this order all regions of momenta (hard, soft, and ultrasoft) contribute to the energy.

We would like to emphasize that the procedure we propose for higher order corrections to the positronium (and other QED bound states) is totally systematic. It uses two EFTs, namely, NRQED and pNRQED. Both the matching from QED to NRQED and from NRQED to pNRQED can be done order by order in $1 / m$ and $\alpha$, and static propagators for the fermions can be used. This together with the use of dimensional regularization simplifies a lot the calculations. The actual bound state calculation is done at the level of pNRQED for the wave function field and is very similar to a standard quantum mechanical calculation, the only difference being that the wave function field couples to US photons in a field theoretical fashion.

We believe that the clarity and simplicity of this formalism will allow one to carry out higher order bound state calculations in QED very efficiently. In order to illustrate this point let us pose ourselves the calculation of the positronium spectrum at $O\left(m \alpha^{6}\right)$ and see the extra calculations required in order to obtain the pNRQED Lagrangian at this order. Clearly all contributions that we obtain at $O\left(m \alpha^{5}\right)$ which are multiplied by a Wilson coefficient will give a contribution at $O\left(m \alpha^{6}\right)$ by just calculating the Wilson coefficient to next order in $\alpha$. This requires the matching from
QED to NRQED at two loops. The relativistic correction to the kinetic energy, $O\left(1 / \mathrm{m}^{5}\right)$, in NRQED should be kept. Terms $O\left(1 / \mathrm{m}^{4}\right)$ in the NRQED Lagrangian would now contribute to the potential but they do so only at the tree level. Hence their Wilson coefficients are only necessary at the tree level. Those for the bilinear terms may be obtained from [20] whereas those for the four-fermion operators from [21]. Terms $O\left(1 / \mathrm{m}^{3}\right)$ also contribute at the tree level and may contribute at one loop. In either case the Wilson coefficients are only needed at the tree level which are known [14]. In addition $O\left(1 / \mathrm{m}^{2}\right)$ terms in the NRQED Lagrangian would now contribute to the potential at one loop and $O(1 / \mathrm{m})$ terms at two loops. It is also easy to see by inspecting the next order terms of the multipole expansion in the pNRQED Lagrangian that, due to angular momentum conservation, no contribution of US photons arises at $O\left(m \alpha^{6}\right)$. There would only be a new qualitative feature, namely, that time derivatives multiplying potential terms would arise (for instance, from the expansion of the energy in the one-transversephoton exchange at the tree level). This time derivatives can be disposed of by using the equations of motion in pNRQED (now with potential terms included) as has been done in Appendix A, according to the philosophy of Ref. [16] (see also [17]). This calculation would produce an independent check of the existing results obtained very recently in Ref. [22].

It is important to be able to calculate systematically higher orders in QED as a test of the standard model in a sector where QCD does not play any relevant role. Any signal of new physics here should be much clearer than in the hadronic sector as, for instance, in orthopositronium decay where there seem to be some difficulties in explaining the data (see [23] and references therein). ${ }^{6}$

Finally, we would like to stress that the idea of separating the calculation of the binding energy (or any other observable) of a nonrelativistic bound state system in three stages, namely, (i) integrating out the hard scale, (ii) integrating out the soft scale, and (iii) calculating the bound state energy

\footnotetext{
${ }^{6}$ Only one experiment [24] seems to be compatible with theoretical predictions.
} 

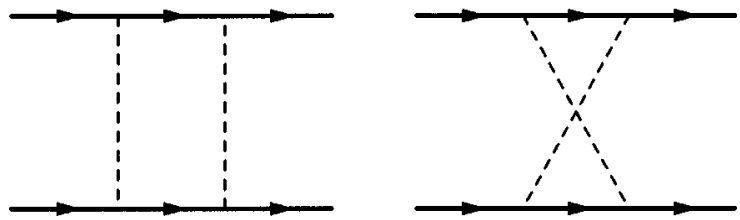

FIG. 4. The nonzero relevant diagrams for the matching at one loop in the Feynman gauge setting $E$ to zero and with no $1 / m$ insertions.

when only ultrasoft degrees of freedom are present, is neither confined to positronium nor to the QED realm. Indeed, we have already shown in Ref. [11] that it can be applied to hydrogenlike atoms, and it should be easy to work out pNRQED for muonium, dimuonium, and other two-body QED bound states. In particular, pionium, a QED bound state, which, however, decays strongly, has received considerable attention lately [25]. Its decay width turns out to be proportional to the pion scattering length, which is an essential input to fix the parameters of the chiral Lagrangian [26]. In order to extract the scattering length neatly from the experimental data a good control on the electromagnetic corrections is necessary. pNRQED for pionium can definitely help in that goal. Beyond QED, heavy quarkonium systems also form nonrelativistic bound states. We have already proposed that potential NRQCD (pNRQCD), an EFT for NRQCD analogous to pNRQED for NRQED, should be useful to study these systems [10]. The techniques presented here may also help in the understanding of nucleon-nucleon bound states from the heavy baryon chiral Lagrangian, which have also received quite some attention during the last years [27].

\section{ACKNOWLEDGMENTS}

Financial support from CICYT, contract AEN95-0590, and from CIRIT, contract GRQ93-1047, is acknowledged. A.P. acknowledges financial support from the TMR network under contract FMRX-CT96-0008.

\section{APPENDIX A: $O\left(m \alpha^{4}\right)$ MATCHING IN THE FEYNMAN GAUGE}

In this appendix we check that the pNRQED Lagrangian is gauge independent at $O\left(m \alpha^{4}\right)$ once it is written in the standard form. By the standard form we understand that all time derivatives in higher order terms are disposed of by the use of equations of motion or, alternatively, by local field redefinitions [14,16] (see also [17]). In order to do so, the matching calculation is redone in the Feynman gauge.

The main difference between the Coulomb gauge and the Feynman gauge, as far as the matching of the four-point Green function is concerned, is that, in the former, loop diagrams involving $A_{0}$ only can be set to zero in NRQED because there is no scale for integration over the energy, whereas in the latter they must be kept because the poles of the $A_{0}$ propagator now relate energy and momentum.

The counting in Sec. II implies now that the following extra diagrams must be considered: (i) the one-loop dia-

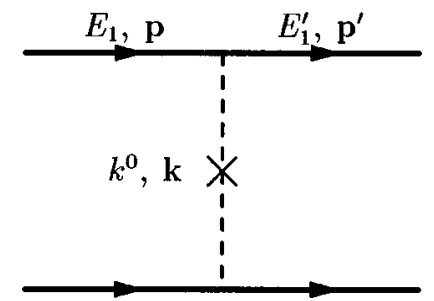

FIG. 5. Correction to the $A_{0}$ propagator due to energy insertions in the Feynman gauge.

grams of Fig. $4\left[O\left(m \alpha^{3}\right)\right]$, (ii) the same diagrams with a $\mathbf{p}^{2} / 2 m$ insertion in either fermionic line $\left[O\left(m \alpha^{4}\right)\right]$, (iii) the same diagrams with external energy insertions arising from the expansion of the propagators about zero external energy $\left[O\left(m \alpha^{4}\right)\right]$, and (iv) two-loop diagrams involving $A_{0}$ propagators only $\left[O\left(m \alpha^{4}\right)\right]$.

It is easy to see that diagrams (i) cancel each other. Diagrams (ii) and (iii) vanish individually, essentially because they have an odd number of static propagators. Diagrams (iv) have already been seen to cancel in Ref. [28]. Then we are left with the same diagrams we had in the Coulomb gauge [all diagrams in Fig. 1, except (d) which is $O\left(m \alpha^{5}\right)$ ], but now they must be calculated in the Feynman gauge.

In fact, all the diagrams give the same result except Figs. 1a and 1e. The latter now reads

$$
\tilde{V}_{\text {tree }}^{(e)}(\text { Feynman })=\tilde{V}_{\text {tree }}^{(e)}(\text { Coulomb })-\frac{\pi \alpha}{m^{2}} \frac{\left(\mathbf{p}^{2}-\mathbf{p}^{\prime 2}\right)^{2}}{\mathbf{k}^{4}} .
$$

Figure 1a now receives a contribution due to the expansion of the external energies about zero in the $A_{0}$ propagator, which we depicted in Fig. 5. It reads

$$
\widetilde{V}_{\text {tree }}^{\text {new }}(\text { Feynman })=-4 \pi \alpha\left(\frac{k^{0}}{\mathbf{k}^{2}}\right)^{2},
$$

where $k^{0}=E_{1}-E_{1}^{\prime}$. Here $E_{1}$ and $E_{1}^{\prime}$ give rise to time derivatives in the pNRQED Lagrangian:

$$
\begin{aligned}
L^{\text {new }}(\text { Feynman })= & -\int d^{3} \mathbf{x}_{1} d^{3} \mathbf{x}_{2} \partial_{0}^{2}\left[\psi^{\dagger} \psi\left(t, \mathbf{x}_{1}\right)\right] \\
& \times \int \frac{d \mathbf{k}}{(2 \pi)^{3}} e^{i \mathbf{k} \cdot \mathbf{x}} \frac{1}{\mathbf{k}^{4}} \chi_{c}^{\dagger} \chi_{c}\left(t, \mathbf{x}_{2}\right) \\
= & \int d^{3} \mathbf{x}_{1} d^{3} \mathbf{x}_{2} \partial_{0}\left[\psi^{\dagger} \psi\left(t, \mathbf{x}_{1}\right)\right] \\
& \times \int \frac{d \mathbf{k}}{(2 \pi)^{3}} e^{i \mathbf{k} \cdot \mathbf{x}} \frac{1}{\mathbf{k}^{4}} \partial_{0}\left[\chi_{c}^{\dagger} \chi_{c}\left(t, \mathbf{x}_{2}\right)\right] .
\end{aligned}
$$

We can get rid of these derivatives by using the equations of motion. Notice, however, that now potential terms enter in 
the equations of motion. Their explicit inclusion can be avoided in this case by using the continuity equation in the last equality of Eq. (A3):

$$
\frac{\partial \rho}{\partial t}+\nabla \cdot \mathbf{j}=0
$$

where $\rho=\psi^{\dagger} \psi$ and

$$
\mathbf{j}=-\frac{i}{2 m}\left[\psi^{\dagger} \nabla \psi-\left(\nabla \psi^{\dagger}\right) \psi\right]
$$

Equation (A2) can now be written as

$$
\tilde{V}_{\text {tree }}^{\text {new }}(\text { Feynman })=+\frac{\pi \alpha}{m^{2}} \frac{\left(\mathbf{p}^{2}-\mathbf{p}^{\prime 2}\right)^{2}}{\mathbf{k}^{4}},
$$

which just cancels the extra contribution in Eq. (A1). We have then proved that the pNRQED Lagrangian written in the standard form (i.e., with no time derivatives) at $O\left(m \alpha^{4}\right)$ is exactly the same in the Coulomb and Feynman gauges. Notice that it has been crucial to write the time derivatives in a symmetric fashion in order to use the continuity equation. The naive use of the on-shell condition $k^{0}=\mathbf{p}^{2} / 2 m-\mathbf{p}^{\prime 2} / 2 m$ in Eq. (A2) leads to incorrect results.

\section{APPENDIX B: pNRQED LAGRANGIAN FOR THE UNEQUAL MASS CASE}

Here we display the Lagrangian relevant for the calculation of the mass to $O\left(m \alpha^{5}\right)$ for the unequal mass case (we assume $\left.m_{1}, m_{2} \gg \mathbf{p} \gg E\right)$. The charge of each particle has opposite sign:

$$
\begin{aligned}
L_{\mathrm{PNRQED}}= & \int d^{3} \mathbf{x} d^{3} \mathbf{X} d t S^{\dagger}(\mathbf{x}, \mathbf{X}, t)\left\{i \partial_{0}-\frac{\mathbf{p}^{2}}{\mu_{12}}+\frac{\mathbf{p}^{4}}{8 m_{1}^{3}}+\frac{\mathbf{p}^{4}}{8 m_{2}^{3}}-\frac{\mathbf{P}^{2}}{2 M}-V\left(\mathbf{x}, \mathbf{p}, \boldsymbol{\sigma}_{1}, \boldsymbol{\sigma}_{2}\right)+e \mathbf{x} \cdot \mathbf{E}(\mathbf{X}, t)\right\} S(\mathbf{x}, \mathbf{X}, t) \\
& -\int d^{3} \mathbf{x} \frac{1}{4} F_{\mu \nu} F^{\mu \nu},
\end{aligned}
$$

where $M=m_{1}+m_{2}, \mu_{12}=m_{1} m_{2} /\left(m_{1}+m_{2}\right), \mathbf{X}$ and $\mathbf{X}$, and $\mathbf{p}$ and $\mathbf{P}$ are the relative and center-of-mass coordinate and momentum, respectively. The potential now reads

$$
\begin{aligned}
V= & -\frac{\alpha}{|\mathbf{x}|}-\frac{\alpha}{2 m_{1} m_{2}} \frac{1}{|\mathbf{x}|}\left(\mathbf{p}^{2}+\frac{1}{\mathbf{x}^{2}} \mathbf{x} \cdot(\mathbf{x} \cdot \mathbf{p}) \mathbf{p}\right)+\frac{\delta^{(3)}(\mathbf{x})}{m_{1} m_{2}}\left[\pi \alpha\left(\frac{c_{D}^{(2)} m_{1}^{2}+c_{D}^{(1)} m_{2}^{2}}{2 m_{1} m_{2}}-2 c_{F}^{2}\right)+d_{s}+3 d_{v}\right. \\
& \left.-16 \pi \alpha d_{2}\left(\frac{m_{1}^{2}+m_{2}^{2}}{m_{1} m_{2}}\right)+\frac{\alpha^{2}}{3}-\frac{7 \alpha^{2}}{3} \log \mu^{2}\right]-\frac{7 \alpha^{2}}{6 \pi m_{1} m_{2}} \operatorname{reg} \frac{1}{|\mathbf{x}|^{3}}+\frac{\delta^{(3)}(\mathbf{x})}{m_{1} m_{2}} \mathbf{S}^{2}\left(\pi \alpha \frac{4}{3} c_{F}^{2}-2 d_{v}\right) \\
& +\frac{\alpha c_{F}}{m_{1} m_{2}} \frac{1}{|\mathbf{x}|^{3}} \mathbf{L} \cdot \mathbf{S}+\frac{\alpha c_{S}}{2 m_{1} m_{2}} \frac{1}{|\mathbf{x}|^{3}} \mathbf{L} \cdot\left(\frac{\mathbf{s}_{2} m_{1}^{2}+\mathbf{s}_{1} m_{2}^{2}}{m_{1} m_{2}}\right)+\frac{\alpha c_{F}^{2}}{4 m_{1} m_{2}} \frac{1}{|\mathbf{x}|^{3}} S_{12}(\hat{\mathbf{x}}),
\end{aligned}
$$

where

$$
c_{D}^{(i)}=1+\frac{\alpha}{\pi}\left(\frac{4}{3} \log \frac{m_{i}^{2}}{\mu^{2}}\right),
$$

and now $d_{s}$ and $d_{v}$ read (see [10])

$$
\begin{gathered}
d_{s}=-\frac{\alpha^{2}}{m_{1}^{2}-m_{2}^{2}}\left\{m_{1}^{2}\left(\log \frac{m_{2}^{2}}{\mu^{2}}+\frac{1}{3}\right)-m_{2}^{2}\left(\log \frac{m_{1}^{2}}{\mu^{2}}+\frac{1}{3}\right)\right\}, \\
d_{v}=\frac{\alpha^{2}}{m_{1}^{2}-m_{2}^{2}} m_{1} m_{2} \log \frac{m_{1}^{2}}{m_{2}^{2}} .
\end{gathered}
$$

The Lagrangian (B2) must be corrected if there are charged particles of masses $m_{i}, i=3,4 \ldots$, similar or smaller than $m:=\max \left\{m_{1}, m_{2}\right\}$. Each particle of mass $m_{i}$ such that $m \gtrsim m_{i} \gg \mu_{12} \alpha$ gives an extra contribution $1 / m_{i}^{2}$ multiplying to $d_{2}$ in Eq. (B2). Each particle of mass $\mu_{12} \alpha \gtrsim m_{i} \gg \mu_{12} \alpha^{2}$ gives extra nontrivial contributions to the potential [25].

[1] W. E. Caswell and G. P. Lepage, Phys. Lett. 167B, 437 (1986).

[2] T. Kinoshita and M. Nio, Phys. Rev. D 53, 4909 (1996).

[3] P. Labelle, S. M. Zebarjad, and C. P. Burgess, Phys. Rev. D 56, 8053 (1997).
[4] A. H. Hoang, P. Labelle, and S. M. Zebarjad, Phys. Rev. Lett. 79, 3387 (1997).

[5] H. A. Bethe and E. E. Salpeter, Phys. Rev. 82, 309 (1951).

[6] P. Labelle, Phys. Rev. D 58, 093013 (1998). 
[7] M. Luke and A. V. Manohar, Phys. Rev. D 55, 4129 (1997).

[8] B. Grinstein and I. Z. Rothstein, Phys. Rev. D 57, 78 (1998).

[9] M. Luke and M. J. Savage, Phys. Rev. D 57, 413 (1998).

[10] A. Pineda and J. Soto, Nucl. Phys. B (Proc. Suppl.) 64, 428 (1998).

[11] A. Pineda and J. Soto, Phys. Lett. B 420, 391 (1998).

[12] M. Beneke and V. A. Smirnov, Nucl. Phys. B522, 321 (1998).

[13] H. W. Griesshammer, "The Soft Regime in NRQCD," Report No. NT@UW-98-12, hep-ph/9804251.

[14] A. V. Manohar, Phys. Rev. D 56, 230 (1997).

[15] A. Pineda and J. Soto, Phys. Rev. D (to be published), hep-ph/9802365.

[16] S. Scherer and H. W. Fearing, Phys. Rev. D 52, 6445 (1995).

[17] C. Balzereit, "Renormalizing Heavy Quark Effective Theory at $O\left(1 / m_{Q}^{3}\right)$, ,' hep-ph/9801436.

[18] S. Titard and F. J. Ynduráin, Phys. Rev. D 49, 6007 (1994).

[19] S. N. Gupta, W. W. Repko, and C. J. Suchyta III, Phys. Rev. D 40, 4100 (1989).

[20] S. Balk, A. Ilakovac, J. C. Körner, and D. Pirjol, Ahrenshoop
Symposium 1993, Report No. QCD161:S973:1993 (unpublished), pp. 315-326.

[21] G. T. Bodwin, E. Braaten, and G. P. Lepage, Phys. Rev. D 51, 1125 (1995); 55, 5853(E) (1997).

[22] K. Pachucki and S. G. Karshenboim, Phys. Rev. Lett. 80, 2101 (1998).

[23] P. Labelle, G. P. Lepage, and U. Magnea, Phys. Rev. Lett. 72, 2006 (1994).

[24] S. Asai, S. Orita, and N. Shinohara, Phys. Lett. B 357, 475 (1995).

[25] P. Labelle and K. Buckley, "A new order $\alpha$ correction to the decay rate of pionium," hep-ph/9804201.

[26] J. Gasser and H. Leutwyler, Ann. Phys. (N.Y.) 158, 142 (1984); Nucl. Phys. B250, 465 (1985).

[27] S. Weinberg, Phys. Lett. B 251, 288 (1990); Nucl. Phys. B363, 3 (1992); C. Ordóñez, L. Ray, and U. van Kolck, Phys. Rev. Lett. 72, 1982 (1994); Phys. Rev. C 53, 2086 (1996); D. B. Kaplan, M. J. Savage, and M. B. Wise, nucl-th/9802075.

[28] M. Peter, Phys. Rev. Lett. 78, 602 (1997); Nucl. Phys. B501, 471 (1997). 